\title{
Embase Session Results
}

No. Query

Results

\#6 \#1 AND \#5

\#5 \#2 OR \#3 OR \#4

$1,388,654$

\#4 'injection'/exp OR injection

616,949

\#3 'parenteral drug administration'/exp OR parenteral AND drug AND administration

\#2 'subcutaneous drug administration'/exp OR subcutaneous AND drug AND administration

\#1 'rheumatoid arthritis'/exp OR 'arthritis deformans' OR 'arthritis, rheumatoid' OR 'arthrosis deformans' OR 'beauvais disease' OR 'chronic polyarthritis' OR 'chronic progressive poly arthritis' OR 'chronic progressive polyarthritis' OR 'chronic rheumatoid arthritis' OR 'disease, beauvais' OR 'infantile rheumatoid arthritis' OR 'inflammatory arthritis' OR 'polyarthritis, primary chronic' OR 'primary chronic polyarthritis' OR 'progressive polyarthritis, chronic' OR 'rheumarthritis' OR

'rheumatic arthritis' OR 'rheumatic polyarthritis' OR 'rheumatism, chronic articular' OR 'rheumatoid arthritis' AND ('methotrexate'/exp OR '4 amino 10 methylfolic acid' OR '4 amino 10 methylpteroylglutamic acid' OR '4 amino n10 methylpteroylglutamic acid' OR 'mtx' OR 'a methopterine' OR 'abitrexate' OR 'amethopterin' OR 'amethopterine' OR 'ametopterine' OR 'antifolan' OR 'biotrexate' OR 'canceren' OR 'cl 14377' OR 'cl14377' OR 'emtexate' OR 'emthexat' OR 'emthexate' OR 'emtrexate' OR 'enthexate' OR 'farmitrexat'

OR 'farmitrexate' OR 'farmotrex' OR 'folex' OR 'folex pfs' OR 'ifamet' OR 'imeth' OR 'intradose mtx' OR 'lantarel' OR 'ledertrexate'

OR 'maxtrex' OR 'metex' OR 'methoblastin' OR 'methohexate' OR 'methotrate' OR 'methotrexat' OR 'methotrexat ebewe' OR 'methotrexate' OR 'methotrexate Ipf' OR 'methotrexate preservative free' OR 'methotrexate sodium' OR 'methotrexate sodium preservative free' OR 'methotrexato' OR 'methoxtrexate' OR 'methrotrexate' OR 'methylaminopterin' OR 'methylaminopterine' OR

'meticil' OR 'metoject' OR 'metothrexate' OR 'metothrexate sodium' OR 'metotrexat' OR 'metotrexate' OR 'metotrexin' OR 'metrex' OR 'mexate' OR 'mexate-aq' OR 'mexate-aq preserved' OR 'mpi 5004' OR 'mpi5004' OR 'n [4 [(2, 4 diamino 6 pteridylmethyl)

methylamino] benzoyl] glutamic acid' OR 'neotrexate' OR 'nordimet' OR 'novatrex' OR 'nsc 740' OR 'nsc740' OR 'otrexup' OR 'rasuvo' OR 'reumatrex' OR 'rheumatrex' OR 'rheumatrex dose pack' OR 'sodium methotrexate' OR 'texate' OR 'texate-t' OR 'texorate' OR 'trexall' OR 'xaken' OR 'zexate') AND ('oral drug administration'/exp OR 'administration, oral' OR 'drug administration, oral' OR 'oral administration' OR 'oral drug administration' OR 'oral drug intake' OR 'p.o. administration' OR 'p.o. dosage' OR 'p.o. dose' OR 'p.o. drug administration' OR 'p.o. drug intake' OR 'per os drug administration') 